\title{
Bone marrow endothelial cell-derived interleukin-4 contributes to thrombocytopenia in acute myeloid leukemia
}

\begin{abstract}
Ai Gao, ${ }^{1,2, \#}$ Yuemin Gong, 1,2,3,\# Caiying Zhu, ${ }^{1,2}$ Wanzhu Yang, ${ }^{1,2}$ Qing Li,, ${ }^{1,2}$ Mei Zhao,,$^{1,2}$ Shihui Ma, ${ }^{1,2}$ Jianyong $\mathrm{Li}^{3}{ }^{3}$ Sha Hao, ${ }^{1,2,4,5,{ }^{*}}$ Hui Cheng ${ }^{1,2,4,5,{ }^{*}}$ and Tao Cheng $^{1,2,4,5}$,

${ }^{1}$ State Key Laboratory of Experimental Hematology; ${ }^{2}$ Institute of Hematology and Blood Disease Hospital, Chinese Academy of Medical Sciences and Peking Union Medical College, Tianjin; ${ }^{3}$ Department of Hematology, the First Affiliated Hospital of Nanjing Medical University, Jiangsu Province Hospital, Jiangsu; ${ }^{4}$ Center for Stem Cell Medicine, Chinese Academy of Medical Sciences, Tianjin and ${ }^{5}$ Department of Stem Cell \& Regenerative Medicine, Peking Union Medical College, Tianjin, China
\end{abstract}

\#These authors contributed equally to this work.

\section{ABSTRACT}

$\mathrm{N}$ ormal hematopoiesis can be disrupted by the leukemic bone marrow microenvironment, which leads to cytopenia-associated symptoms including anemia, hemorrhage and infection. Thrombocytopenia is a major and sometimes fatal complication in patients with acute leukemia. However, the mechanisms underlying defective thrombopoiesis in leukemia have not been fully elucidated. In the steady state, platelets are continuously produced by megakaryocytes. Using an MLL-AF9-induced acute myeloid leukemia mouse model, we demonstrated a preserved number and proportion of megakaryocyteprimed hematopoietic stem cell subsets, but weakened megakaryocytic differentiation via both canonical and non-canonical routes. This primarily accounted for the dramatic reduction of megakaryocytic progenitors observed in acute myeloid leukemia bone marrow and a severe disruption of the maturation of megakaryocytes. Additionally, we discovered overproduction of interleukin-4 from bone marrow endothelial cells in acute myeloid leukemia and observed inhibitory effects of interleukin-4 throughout the process of megakaryopoiesis in vivo. Furthermore, we observed that inhibition of interleukin-4 in combination with induction chemotherapy not only promoted recovery of platelet counts, but also prolonged the duration of remission in our acute myeloid leukemia mouse model. Our study elucidates a new link between interleukin-4 signaling and defective megakaryopoiesis in acute myeloid leukemia bone marrow, thereby offering a potential therapeutic target in acute myeloid leukemia.

\section{Introduction}

Historical data have revealed that the majority of patients with leukemia die of cytopenia-associated complications including infection and hemorrhage. ${ }^{1,2}$ Progressive suppression of normal hematopoiesis is the major cause of cytopenias in leukemias and other malignancies. ${ }^{3}$ Normal hematopoiesis is more seriously compromised than leukemic hematopoiesis in acute myeloid leukemia (AML), even with a low leukemia cell burden. ${ }^{4}$ Maintenance of normal hematopoietic homeostasis depends on the bone marrow (BM) microenvironment, which is the site where hematopoietic stem cells (HSC) reside and are regulated through intercellular contacts and signaling molecules. ${ }^{5}$ Interactions between leukemic cells and the BM microenvironment are attracting intense attention. Studies have shown that leukemia causes significant changes in a variety of cells and cytokines in the BM microenvironment, which impair the function of supporting normal 
hematopoiesis., ${ }^{3,6-9}$ Utilizing a congenic AML mouse model, we recently investigated dynamic changes in normal hematopoietic stem and progenitor cell (HSPC) numbers and cell cycling. ${ }^{10}$ Our findings suggested that the AML microenvironment suppressed proliferation and differentiation of normal HSPC, in line with conclusions from another study based on a xenograft AML model. ${ }^{11}$ Notably, from among all the HSPC subpopulations investigated, megakaryocytic-erythroid progenitors were the most affected. Subsequently, we observed the inhibitory effects of CCL3 from the AML microenvironment on erythropoiesis. ${ }^{12}$ Strikingly, CCL3 acted on megakaryocyticerythroid progenitors rather than HSC, and specifically suppressed erythropoiesis without affecting megakaryopoiesis. ${ }^{12}$ How the path from HSC to megakaryocytes $(\mathrm{MK})$ was influenced by the AML BM microenvironment remains to be defined.

Thrombocytopenia is a major and sometimes even fatal complication of acute leukemia.,13 It has been estimated that thrombocytopenia occurs in approximately $75 \%$ of all leukemia patients, with one-third of them having clinically significant bleeding. ${ }^{14,15}$ In healthy individuals, functional platelets are produced on a daily basis by mature MK, which originate from multipotent HSPC. Thrombopoietin is recognized as the principal regulatory cytokine throughout the process of megakaryopoiesis. ${ }^{16}$ An elegant study by Rauch et al. ${ }^{17}$ attributed thrombocytopenia in AML patients to thrombopoietin scavenging by MPL hi leukemic blasts and proposed the MPL ${ }^{\text {hi }}$ state as an indicator for more severe thrombocytopenia at diagnosis. However, despite its demonstrated effects in alleviating chemotherapy-associated thrombocytopenia in patients with solid tumors, thrombopoietin did not work well for acute leukemia patients who underwent chemotherapy or hematopoietic stem cell transplantation. ${ }^{18}$ These phenomena suggest that the mechanism of thrombocytopenia is much more complicated in AML, and cannot be simply explained by chemotherapyinduced injury or a paucity of thrombopoietin. Thrombocytopenia may be a prolonged state due to the profound influence of leukemia on the process of megakaryopoiesis and thrombopoiesis, and on the BM microenvironment which tightly regulates hematopoiesis. In fact, recent studies have indicated that the perturbation of the BM microenvironment of leukemic hosts cannot be completely cured by current therapies, even if the leukemic burden is substantially reduced.,19 Thus, a thorough analysis of megakaryopoiesis and thrombopoiesis during leukemia development is required to search for detrimental factors in the leukemic microenvironment that could serve as potential therapeutic targets for these processes. In the classical hierarchical model of hematopoiesis, MK and erythrocytes share common progenitors. ${ }^{20}$ However, recent studies suggest that $M K$ can be generated directly by an upstream HSC subpopulation, independently of other lineage fates. ${ }^{21,22}$ The alterations along the path from HSC to MK in AML are still unknown.

In the present study, we used an MLL-AF9-induced AML mouse model to analyze the dynamic changes of HSPC with MK potential. We found that thrombocytopenia in AML was caused primarily by reduced megakaryocytic differentiation of $\mathrm{CD}_{150}{ }^{+} \mathrm{HSC}$ and defective maturation of MK. Furthermore, we detected elevated interleukin-4 (IL-4) levels in AML BM, which were found to inhibit megakaryopoiesis and thrombopoiesis in vivo. Finally, we observed that inhibition of IL-4 combined with chemotherapy attenuated leukemia progression as well as thrombocytopenia in our AML mouse model.

\section{Methods}

\section{Mice}

C57BL/6-Ly5.1 (Ly5.1) and C57BL/6-Ly5.2 (Ly5.2) mice were purchased from the State Key Laboratory of Experimental Hematology (SKLEH). R26-tdTomato mice ${ }^{23}$ were purchased from Jackson Laboratory and actin-eGFP mice ${ }^{24}$ were kindly provided by Dr. Bing Liu (Academy of Military Medical Sciences, Beijing, China). Mice experiments were approved by the Institutional Animal Care and Use Committee of SKLEH.

\section{Flow cytometry}

Details of the staining and enrichment procedures for flow cytometry have been described previously. ${ }^{25}$ Intracellular von Willebrand factor (vWF) and Ki67 staining was performed using a BD IntraSure ${ }^{\mathrm{TM}}$ Kit (BD Biosciences, Franklin Lakes, NJ, USA). Cell surface markers for phenotypic analyses of hematopoietic and niche cells are listed in Online Supplementary Table S1. The antibodies used are listed in Online Supplementary Table S2.

\section{Acquisition of bone marrow niche cells}

Cells were isolated following a previously described protocol. ${ }^{26}$ Immunophenotyping and antibodies are listed in Online Supplementary Table S1 and Online Supplementary Table S2, respectively.

\section{Bone sectioning and immunofluorescence imaging}

Femora were fixed in $4 \%$ paraformaldehyde and dehydrated in $30 \%$ sucrose at $4^{\circ} \mathrm{C}$ prior to being embedded in optimal cutting temperature compound and frozen. For two-dimensional imaging, frozen femora were then cut into $5 \mu \mathrm{m}$ sections using a cryostat and stained according to the Kawamoto method. ${ }^{27}$ Images were acquired using a UltraView VOX confocal microscope (PerkinElmer, Waltham, MA, USA) and analyzed by Volocity.

\section{Enzyme-linked immunosorbent assays}

BM supernatants were prepared by flushing femora and tibiae of leukemic and control mice with $0.5 \mathrm{~mL}$ phosphate-buffered saline. Enzyme-linked immunosorbent assays for IL-4 (R\&D Systems, Minneapolis, MN, USA) were performed according to the manufacturer's instructions. The concentrations were calculated using standard curves.

\section{Isolation of bone marrow megakaryocytes}

BM cells were flushed out and incubated with CD41-APC antibody for $15 \mathrm{~min}$ on ice. CD41+ cells were then enriched using anti-CD41 microbeads and incubated in Dulbecco modified Eagle medium supplemented with $10 \%$ fetal bovine serum and $10 \mu \mathrm{g} / \mathrm{mL}$ Hoechst 33342 at $37^{\circ} \mathrm{C}$ for $1 \mathrm{~h}$. MK were sorted as $\mathrm{SSC}^{\text {high }}$, CD $41^{\text {high }}$, ploidy $\geq 8 \mathrm{~N}$ cells through a $100 \mu \mathrm{m}$ nozzle.

\section{Microarray analysis}

The microarray was performed at Cnkingbio Company (Beijing, China). Gene set enrichment analysis was performed using GSEA v2.2.0. The microarray data have been deposited in the NCBI's Gene Expression Omnibus under accession number GSE112942. 


\section{Statistics}

Unless otherwise stated, data are expressed as mean or mean \pm standard error of the mean as indicated. $P$ values were generated using an unpaired Student $t$-test and analysis of variance. GraphPad Prism and IBM SPSS Statistics software were used for the statistical analyses.

\section{Results}

\section{Megakaryocytic differentiation from hematopoietic stem cells via both canonical and non-canonical routes was markedly inhibited in acute myeloid leukemia bone marrow}

To elucidate the perturbation of the orchestrated process of thrombopoiesis in AML, we used a non-irradiated MLL-AF9-induced AML mouse model (Online Supplementary Figure S1A). ${ }^{10}$ AML mice exhibited a progressive decrease of platelets during leukemia development. On day 14, when leukemic infiltration exceeded $80 \%$ of the whole BM, platelet counts were reduced to $\sim 14 \%$ of the normal level (Online Supplementary Figure $S 1 B)$. Meanwhile, we detected simultaneous loss of BM $\mathrm{MK}$ using a flow cytometry gating strategy introduced by Heazlewood et al. ${ }^{28}$ (Online Supplementary Figure S1C, D). These results suggested that our AML mouse model could mimic the thrombocytopenic process that occurs in patients with AML.

The developmental landscape of BM MK has not been fully understood. It was estimated that approximately half of all $\mathrm{MK}$ progenitors (MkP) were from long-term (LT)-HSC which contributed little to other lineages ${ }^{29}$ in native hematopoiesis, and the other half were from multipotent progenitors (MPP), especially MPP $2 .{ }^{21}$ Thus, we decided to measure the numbers and proportions of these MK-primed subpopulations during the progression of AML (Figure 1A).

For ease of comparison, we normalized the values of the AML group at indicated time points to that of healthy controls (day 0). All of these subpopulations decreased significantly as the leukemia developed, but to different extents. The changes of pre-megakaryocytic-erythroid (PreMegE) and MkP were much more dramatic: at the end stage of AML, the total number of residual PreMegE was less than $1 \%$ of normal level, and $\sim 4.9 \%$ of $\mathrm{MkP}$ were left in leukemic BM (Figure $1 \mathrm{~B}$ and Online Supplementary Figure S2). In contrast, $\sim 17.2 \%$ of MPP2 and $29.6 \%$ of LT-HSC were preserved in AML mice (Figure $1 \mathrm{~B}$ and Online Supplementary Figure S2). Regarding their proportions in CD45.2 normal hematopoietic cells, the proportion of PreMegE dropped substantially and continuously, to about $1 / 10$ of normal level; MkP declined at a moderate rate, to approximately $56 \%$ of that in the healthy state (Figure 1C). In contrast, the proportions of MPP2 and LT-HSC increased progressively $\sim 3.4$-fold and $\sim 5$.9-fold, respectively, as compared to the proportions in healthy controls at day 14 (Figure 1C).

We then assessed the apoptosis and cell cycle state of PreMegE and MkP to determine if the marked decrease of both subsets resulted from excessive cell death or growth arrest. We did not find significant changes in the rate of apoptosis of PreMegE during leukemia progression (Online Supplementary Figure S3A). In addition, their cycling was not inhibited in leukemia BM, and even displayed slightly active cycling in the mid-phase of disease
(Online Supplementary Figure S3B), suggesting their active production of downstream populations. Correspondingly, PreMegE isolated from AML mice at the late stage yielded equal numbers of $\mathrm{CD} 41^{+} \mathrm{MK}$ as the PreMegE isolated from healthy controls (Online Supplementary Figure S3C, D). As far as concerns $\mathrm{MkP}$, their rate of apoptosis even declined in AML (Online Supplementary Figure $S 3 E, F)$, and no significant alteration was detected in their cycling state (Online Supplementary Figure S3G). Additionally, the MK colony-forming capacity of MkP from AML hosts was comparable to that of $\mathrm{MkP}$ from healthy controls when cultured in vitro (Online Supplementary Figure S3H). Since MkP derived from the non-canonical pathway, or shortcut, comprised nearly $70 \%$ of the total $\mathrm{MkP}$ pool, ${ }^{21}$ the severe reduction of their number ( 95\% lost) Figure 1B) and proportion in normal hematopoietic cells ( $44 \%$ lost) (Figure $1 \mathrm{C}$ ) suggests a major defect with the shortcut, or LT-HSC and MPP2.

We then sought to evaluate the MK differentiation potential of LT-HSC and MPP2 in AML BM, which was likely to be reflected by the expression of MK-specific proteins in these subsets. VWF is a protein involved in platelet aggregation and is abundant in $\mathrm{MK}$ and endothelial cells. ${ }^{30}$ It has been reported that $\mathrm{vWF}^{+}$HSC have strikingly higher platelet reconstitution potential than vWF HSC. ${ }^{31}$ We, therefore, evaluated intracellular vWF protein levels in these subsets by flow cytometry using a verified antibody. The proportions of $\mathrm{vWF}^{+}$cells were significantly reduced in both LT-HSC (from $\sim 21.1 \%$ to $~ 13.2 \%$ ) and MPP2 (from $\sim 19.1 \%$ to $~ 5.9 \%$ ) in the late stage of $\mathrm{AML}$ (Figure 2A, B), indicating reduced $\mathrm{MK}$ differentiation from both subsets. Taken together, these data suggest that the scarcity of residual MkP in AML BM was due to severe blockade of $\mathrm{MK}$ differentiation in both the canonical and non-canonical routes.

We then investigated whether the decline of vWF expression indicated a prolonged impairment of the MK potential of MPP2 and LT-HSC. To do this, we used actineGFP mice as AML recipients and isolated eGFP+ $\mathrm{MPP} 2$ and LT-HSC from AML (day 14) or healthy control BM to transplant into sub-lethally irradiated mice. We tracked the reconstitution rates of MPP2 and LT-HSC from day 10 to day 20 for maximum exhibition of donor-derived platelet potential. We observed lower levels of platelet reconstitution of both LT-HSC and MPP2 subsets from AML hosts as early as 14 days after transplantation (Figure 2C). However, the reconstitution of peripheral blood nucleated cells from AML and control groups at that time point showed no difference (Figure 2D). These results suggest that the specific impairment of MK differentiation induced by the AML BM microenvironment would not easily recover in a leukemia-free niche.

\section{Megakaryocyte maturation was severely impaired in acute myeloid leukemia bone marrow}

MkP should experience several cycles of endomitosis and cytoplasmic maturation before they become giant, multinucleated, platelet-producing $M K^{32}$ Endomitosis leads to polyploidization of DNA content, which has been extensively demonstrated to be of vital importance for efficient platelet production. ${ }^{33}$ Of note, it was reported that apoptosis and polyploidization are synchronous and intimately linked events during MK maturation; inhibition of apoptosis delays polyploidization and proplatelet formation. ${ }^{34,35}$ We questioned whether the decreased 
apoptotic rate of $\mathrm{MkP}$ in $\mathrm{AML}$ (Online Supplementary Figure $S 3 F$ ) was linked to a lack of maturation. Thus we assessed the polyploidization of MK in AML BM, and found that $M K$ with high ploidy $(\geq 32 N)$ were reduced to a significantly greater extent than those with low ploidy $(8-16 \mathrm{~N})$ (Figure 3A). As with their proportions in normal hematopoietic cells, the proportion of high-ploidy $\mathrm{MK}$ remained stable whereas the proportions of $8 \mathrm{~N}$ and $16 \mathrm{~N}$ $\mathrm{MK}$ progressively increased, especially the proportion of $8 \mathrm{~N}$ ones (Figure $3 \mathrm{~B}$ ). MK in late-stage AML BM exhibited a clear left shift of ploidy distribution (Figure 3C). To gain further insight into the changes of MK function in AML, we isolated $\mathrm{MK}$ from $\mathrm{AML}$ and healthy control BM for microarray assay (Online Supplementary Figure S4A). The thrombopoietin- $\mathrm{Mpl}$ axis is a main regulator of thrombopoiesis, including MK maturation and platelet production. Our microarray data showed that the levels of $\mathrm{Mpl}$ expression in the two groups were similar (Online Supplementary Figure S4B). Gene expression data (GSE112942) showed downregulation of platelet signature genes and $\alpha$-granule genes in MK from AML (Figure 3D), implying insufficient cytoplasmic maturation. In
A

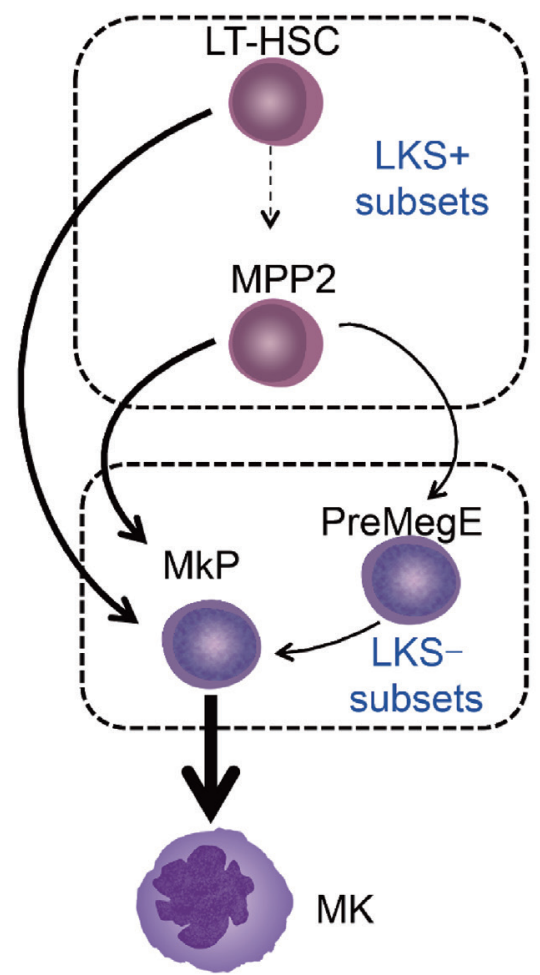

Refined model

B

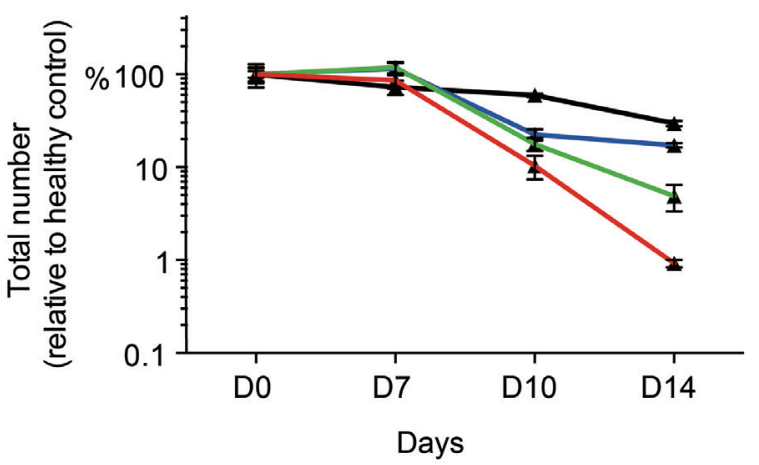

Gated on CD45.1-Lin-CD105-

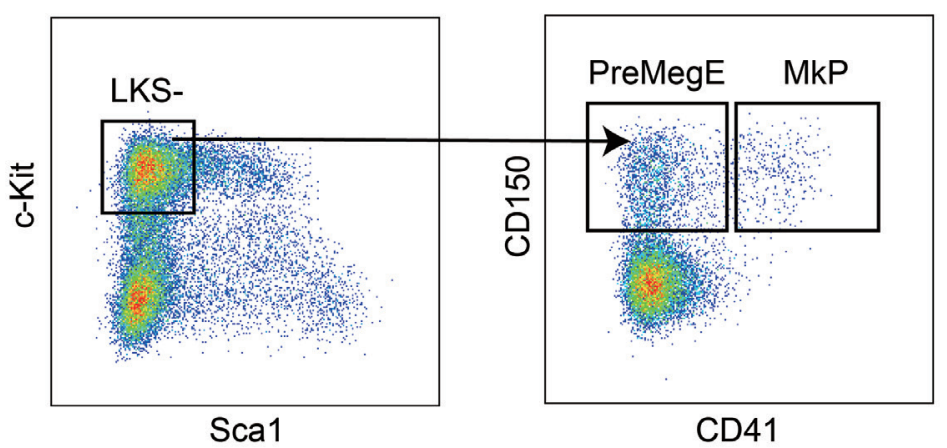

Gated on CD45.1-Lin-

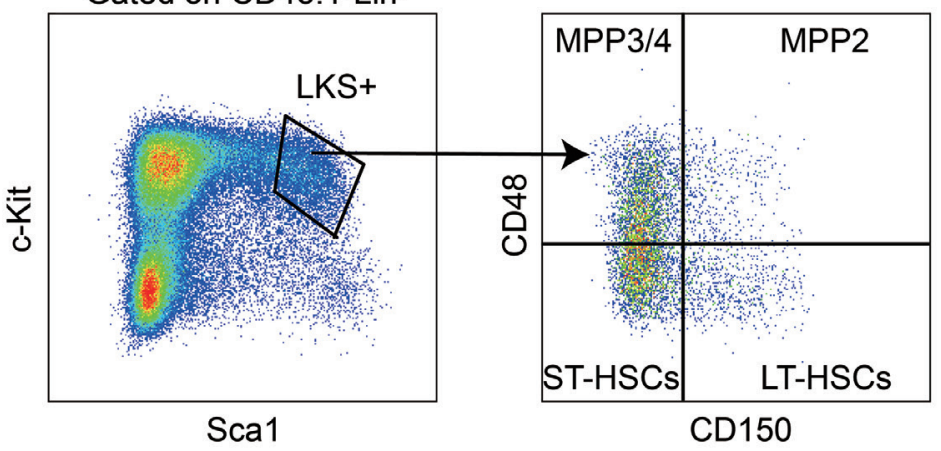

Figure 1. Dynamic changes of hematopoietic stem and progenitor cells with megakaryocyte potential in acute myeloid leukemia. (A) Left, refined model of native megakaryocytic differentiation from the canonical and non-canonical routes. Right, representative flow cytometric plots of hematopoietic stem and progenitor cell subpopulations involved in megakaryocytic differentiation. (B, C) Total numbers (B) and proportions (C) of LT-HSC, MPP2, PreMegE and MkP in normal hematopoietic cells in mice bone marrow at the indicated time points of AML. Data were normalized to the values in healthy controls and are presented as the percentage (B) or fold-increase $(C)$ of controls. Four to five mice for each time point, three independent experiments. Error bars represent the standard error of mean. LT-HSC: longterm hematopoietic stem cells; LKS: Lin /c-Kit/Sca-1+; MPP: multipotent progenitor, PreMegE: pre-megakaryocytic-erythroid; MkP: megakaryocyte progenitor; MK: megakaryocyte; ST-HSC: short-term hematopoietic stem cells. 
addition, apoptosis-associated genes were significantly negatively enriched in MK from AML BM (Figure 3D). These data indicated that the maturation of MK was seriously impaired in AML BM.

The maturation of $M K$ and their platelet-releasing activity have been proven to be dependent on their interaction with vascular endothelium. ${ }^{36}$ We wondered if the defective maturation of MK in AML BM was caused by disrupted interaction with BM endothelial cells. As expected, a variety of genes involved in leukocyte migration, cell adhesion, cytokine-receptor interactions and chemokine signaling pathways were downregulated in MK from AML BM versus control BM (Figure $3 \mathrm{E}, \mathrm{F}$ ), indicating their weakened crosstalk with endothelial cells and the extracellular matrix. We then determined the location of $\mathrm{MK}$ with respect to blood vessels by in situ immunofluorescence imaging. In AML BM, we observed severe destruction of normal vasculature, including lack of typical sinusoid structure and vascular lumens (Figure 3G). Meanwhile, MK in AML BM were located closer to BM endothelial cells (Figure 3G, H), as a result of solid stress applied to them by overgrowing leukemia blasts. The compression of blood vessels and impaired blood perfusion in these areas might reduce the contribution of adjacent $\mathrm{MK}$ to the platelet pool.

Interleukin-4 signaling was upregulated in acute myeloid leukemia bone marrow and exerted inhibitory effects on multiple stages of megakaryocyte differentiation

As thrombopoietin is a key regulator of $\mathrm{MK}$, we examined its concentration in the serum of control and AML mice. Thrombopoietin levels were similar in the two groups (Online Supplementary Figure S5), indicating that thrombopoietin is not the main cause of thrombocytopenia in our AML mouse model. To determine the factors in the leukemia microenvironment that had a negative impact on $M K$ differentiation and maturation, we referred to our cytokine array data of AML versus control BM plasma. ${ }^{12}$ Six cytokines (CCL3, CCL27, IL-4, Tnfrsf1a, Tnfrsf1b and Fcgr1) were upregulated in AML BM plasma. Among them, IL-4 has been reported to inhibit
A

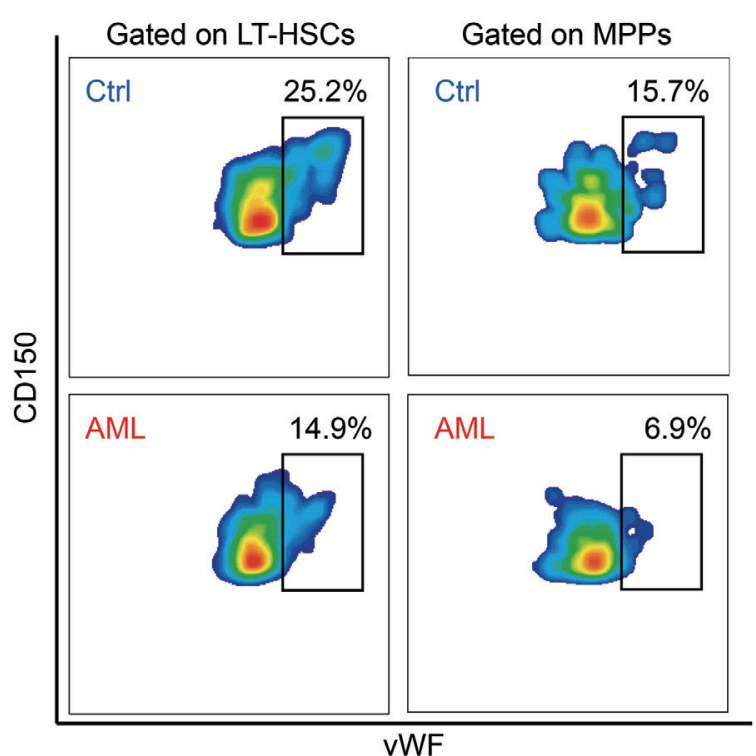

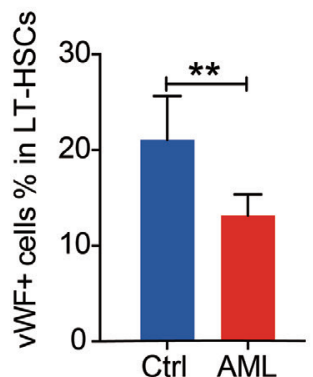

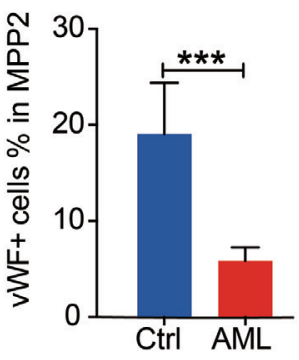

C

LT-HSCs

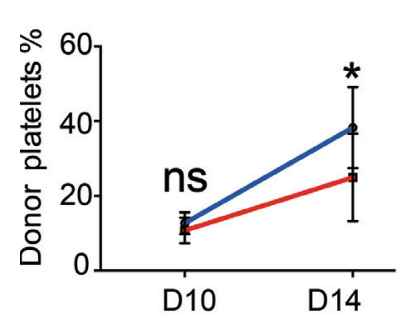

MPP2

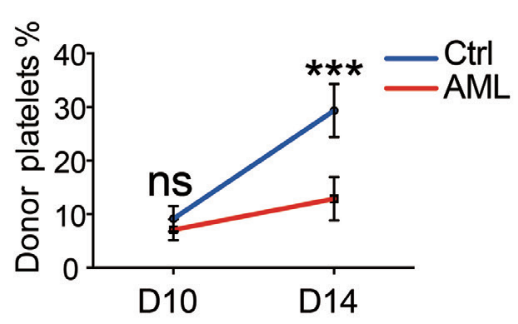

D

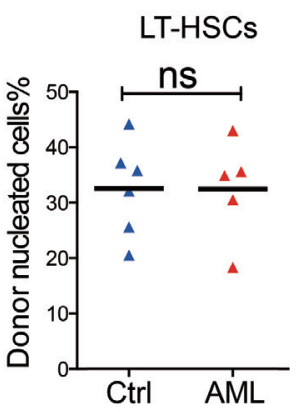

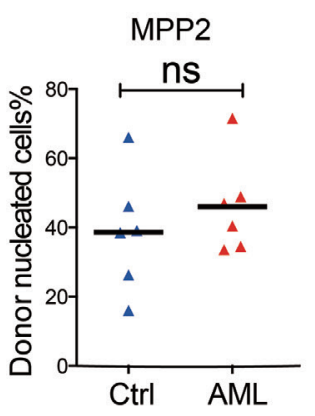

Figure 2. Reduced megakaryocyte differentiation of long-term hematopoietic stem cells and MPP2 from acute myeloid leukemia bone marrow. (A) Representative flow cytometric plots of $\mathrm{VWF}^{+}$cells in LT-HSC and MPP2 from healthy control and acute myeloid leukemia (AML) bone marrow (day 14). (B) Percentage of vWF ${ }^{+}$cells in LT-HSC and MPP2 from healthy control and AML bone marrow. Five to six mice, three independent experiments. (C, D) Percentage of donor-derived eGFP ${ }^{+}$platelets (C) and nucleated cells (D) in peripheral blood on day 14 after transplantation of 400 LT-HSC and 250 MPP2 isolated from control or AML bone marrow (day 14). Six mice, two independent experiments. $* P<0.05, * * P<0.01, * * * P<0.001$. ns, no significant difference. Error bars represent the standard error of mean. vWF: von Willebrand factor; LT-HSC: long-term hematopoietic stem cells; Ctrl: control; MPP: multipotent progenitor; eGFP: enhanced green fluorescent protein. 
A

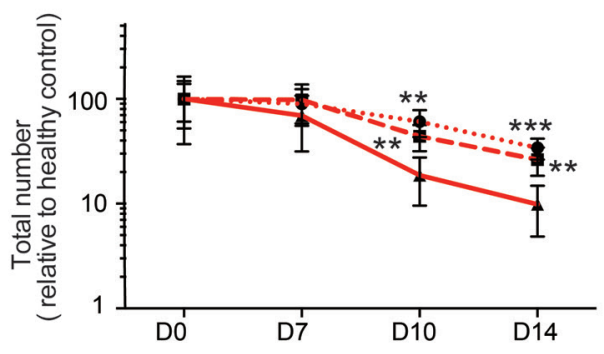

D

Platelet alpha granule

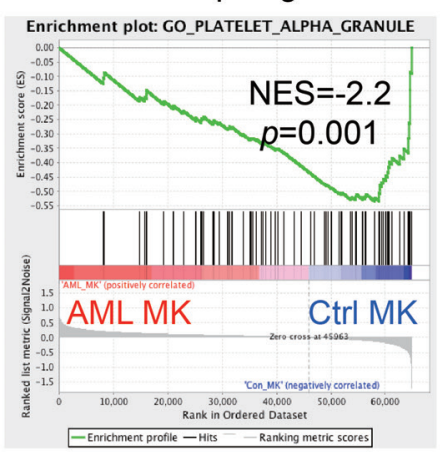

B

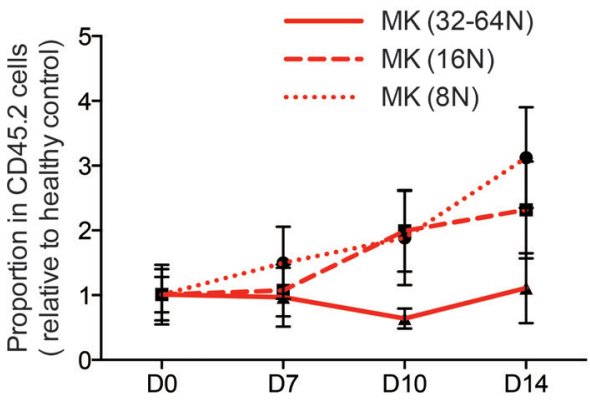

Platelet signature

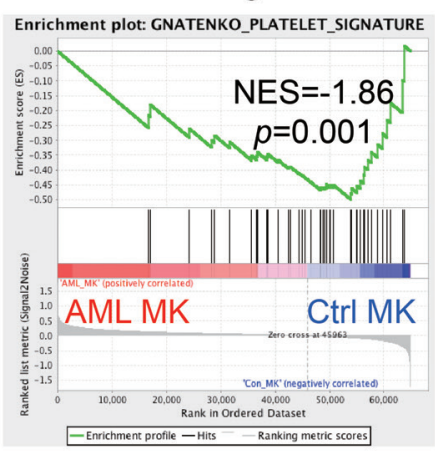

C

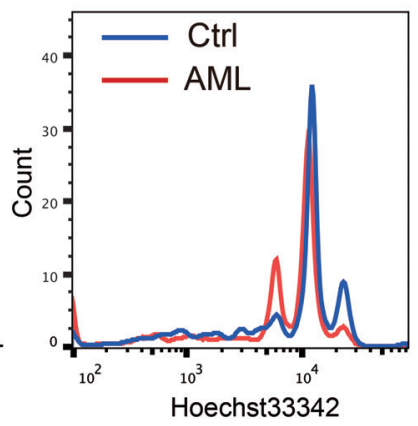

Apoptosis

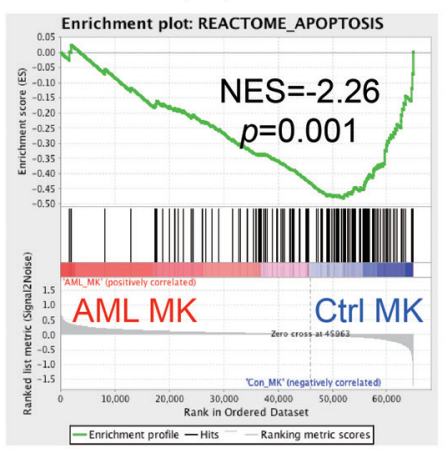

E

F

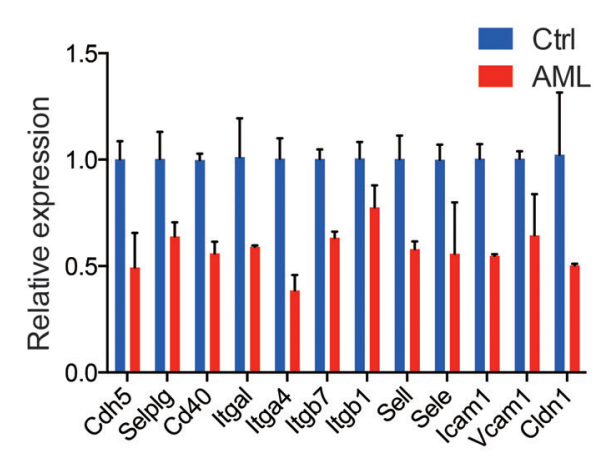

G
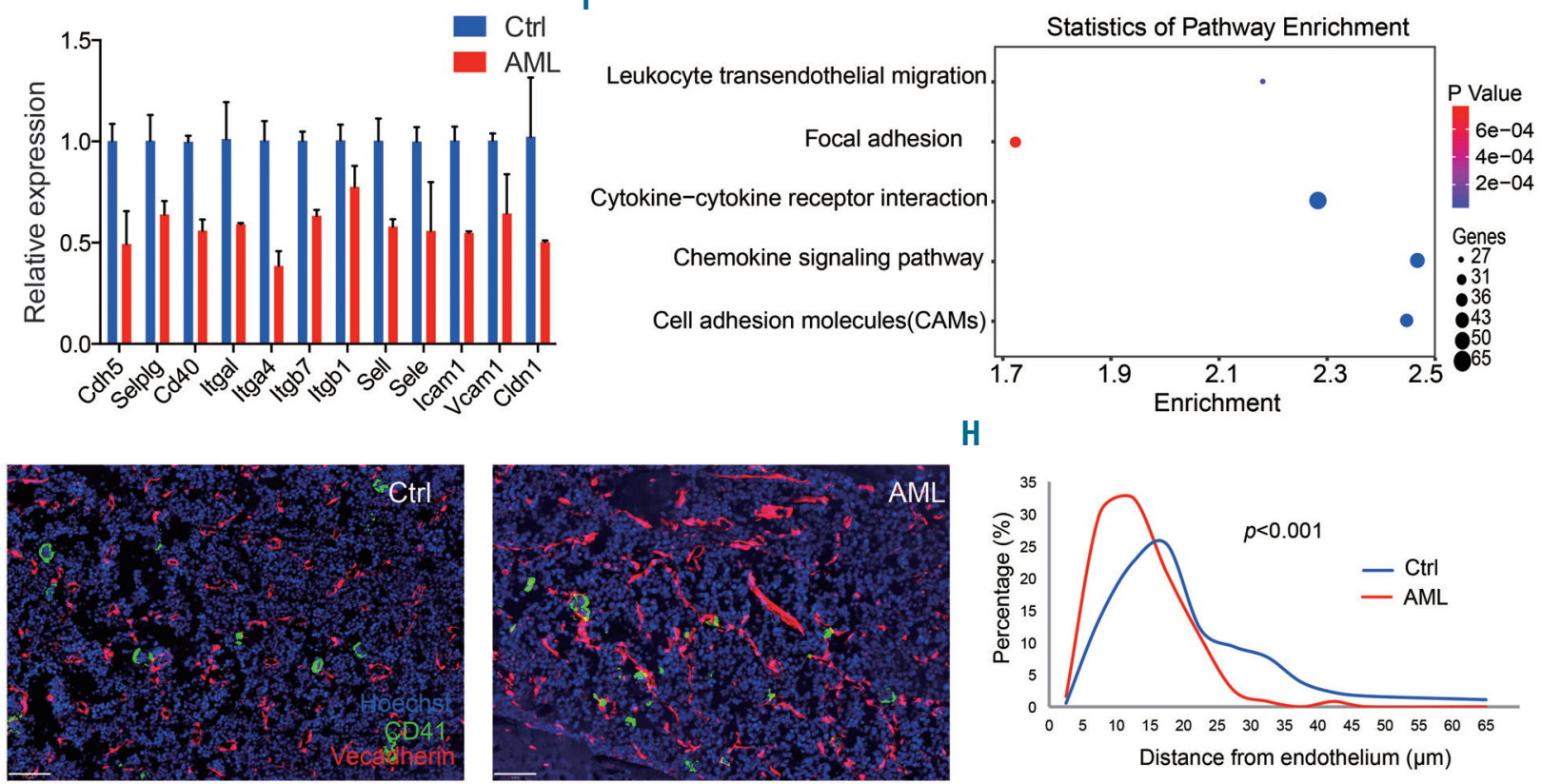

H

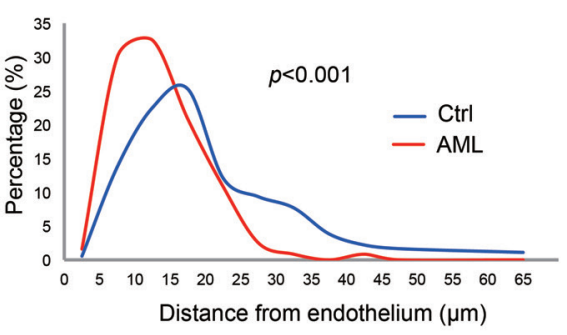

Figure 3. Insufficient maturation of megakaryocytes in acute myeloid leukemia bone marrow. (A) Total numbers of megakaryocytes (MK) with different ploidy in mice bone marrow at indicated time points of the development of acute myeloid leukemia (AML). Data were normalized to the values of healthy controls (Ctrl) and are presented as the percentages of control. Four or five mice for each time point, three independent experiments. (B) Proportions of MK with different ploidy in normal hematopoietic cells in mice bone marrow at the indicated time points of AML. Data were normalized to the values of healthy control and are presented as the folds of control. Four or five for each time point, three independent experiments. (C) Representative flow cytometric plot of MK ploidy distribution in healthy control and AML mice bone marrow (day 14). (D) Negative enrichment of platelet $\alpha$-granule genes, platelet signature genes and apoptosis pathway in AML bone marrowderived MK. NES: normalized enrichment score; $P$, family-wise error rate (FWER) $P$-value. (E) Significantly downregulated $(P<0.05)$ genes encoding adhesion molecules in MK from AML bone marrow. Data are presented as fold-change of gene expression relative to the expression in healthy controls. ( $F$ ) Significant enrichment of downregulated genes in cell-cell or cell-matrix interaction associated KEGG pathways in MK from AML bone marrow. (G) Representative confocal images of CD41+ MK (green) and VE-cadherin ${ }^{+}$vasculature (red) in control and AML (day 14) mice femora. The scale bar represents 70 um. (H) Ouantification of the distance between MK ( $\mathrm{n}=182$ for controls, $\mathrm{n}=120$ for $\mathrm{AML})$ and endothelium. $P$ values were calculated using a two-sample Kolmogorov-Smirnov test. $\star P<0.05, * \star P<0.01$, $* * * P<0.001$. Error bars represent the standard error of mean. 
A

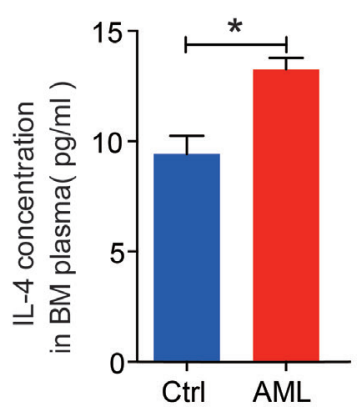

B

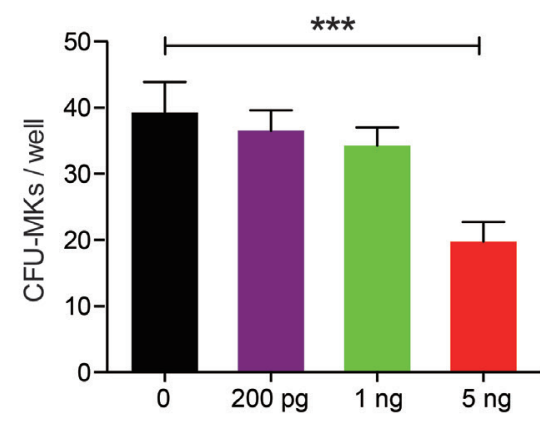

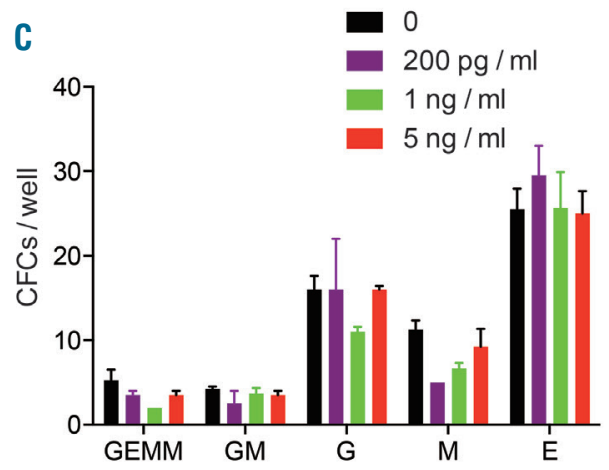

E
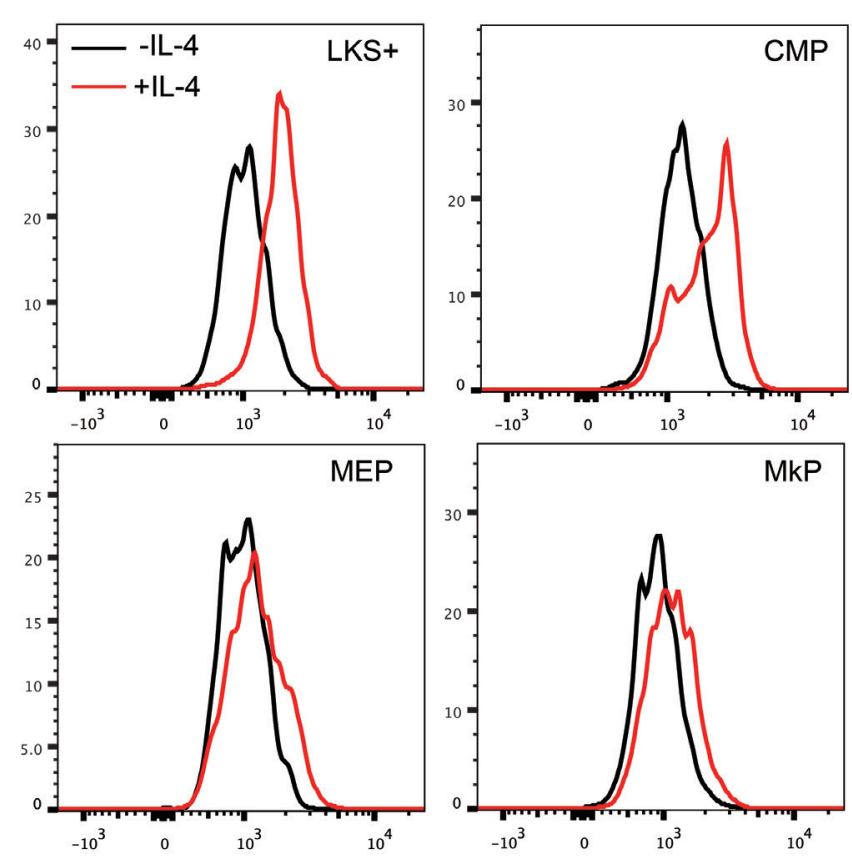

G

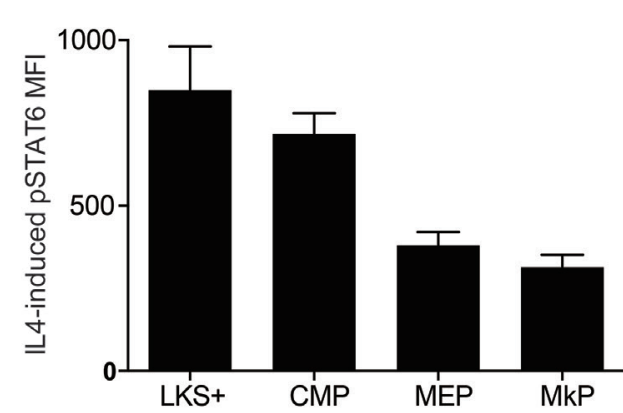

$\mathrm{F}$

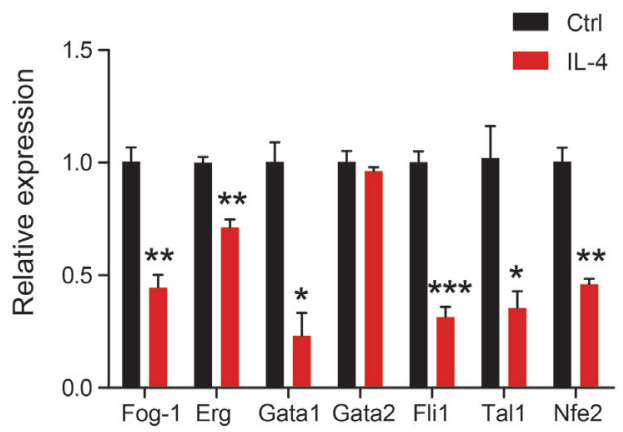

IL-4 signaling

Enrichment plot: PID_IL4_2PATHWAY

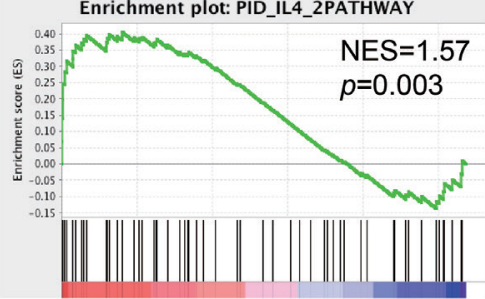

AML LKS+

Ctrl LKS+

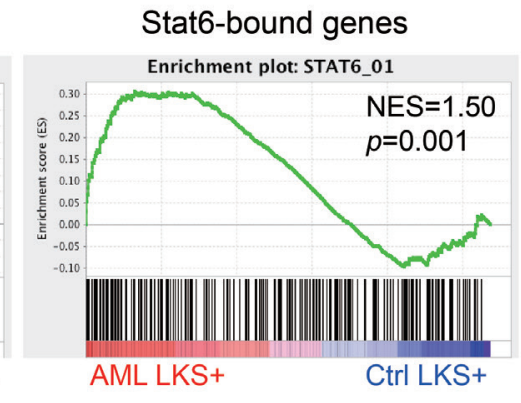

H

pStat6

Figure 4. Elevated interleukin-4 level in acute myeloid leukemia bone marrow. (A) Interleukin-4 (IL-4) protein levels in bone marrow (BM) plasma of control (Ctrl) and acute myeloid leukemia (AML) mice based on enzyme-linked immunosorbent assay measurements. Five mice per group. (B) Number of megakaryocyte colonies (CFU-MK) formed from $5 \times 10^{4}$ whole BM cells incubated with mIL-4 at the indicated concentrations. Four mice, two independent experiments. (C) Number of myeloid and erythroid colonies formed from $1 \times 10^{4}$ whole BM cells incubated with mIL-4 at the indicated concentrations. Four mice, two independent experiments. CFC: colony-forming cells; GEMM: granulocyte, erythrocyte, monocyte, megakaryocyte; GM: granulocyte monocyte; G: granulocyte; M: monocyte; E: erythrocyte. (D) Mean fluorescence intensity (MFI) of phosphorylated Stat6 (pStat6) in FACS-sorted hematopoietic stem and progenitor cells (HSPC) upon exposure to mIL-4 (10 ng/mL) for 20 min, calculated by subtracting the MFI of non-treated controls. Three mice, two independent experiments. LKS: Lin/c-Kit $/$ Sca-1+; MPP: multipotent progenitor, CMP: common myeloid progenitor; MEP, megakaryocyte-erythroid progenitor; MkP: megakaryocyte progenitors. (E) Representative flow cytometric plots of pStat6 in FACS-sorted HSPC upon $\mathrm{mL}-4\left(10 \mathrm{ng} / \mathrm{mL}\right.$ ) exposure for $20 \mathrm{~min}$. (F) Relative expression of megakaryocyte (MK)-associated transcription factors in LKS ${ }^{+}$cells upon mIL-4 (10 ng/mL) exposure for $24 \mathrm{~h}$. Four mice, three independent experiments. (G) Positive enrichment of IL-4 signaling genes and Stat6-bound genes in LKS ${ }^{+}$cells from AML bone marrow. NES: normalized enrichment score; $P$, FWER P-value. (H) Relative expression of IL-4 mRNA in endothelial cells (EC), mesenchymal stem cells $(\mathrm{MSC})$ and osteoblasts $(\mathrm{OBC})$ isolated from $\mathrm{AML}$ and control mice $\mathrm{BM}$. Four or five mice per group, three independent experiments. ${ }^{\prime} P<0.05$, $* \star P<0.01$, $* * * P<0.001$. ns, no significant difference. Error bars represent the standard error of mean. 
megakaryocytic colony formation of human CD34+ BM cells $^{37}$ and to have relevance in the thrombocytopenic state of idiopathic thrombocytopenic purpura and allogeneic hematopoietic stem cell transplantation patients. ${ }^{38,39}$ We confirmed the elevated level of IL-4 in the AML group using enzyme-linked immunosorbent assays (Figure 4A). Our in vitro colony-forming cell assays showed that IL-4 imposed a selective inhibitory effect on colony-forming unit-MK formation from BM cells (Figure 4B) without affecting other myeloid and erythroid lineages (Figure 4C). Interestingly, upon in vitro IL-4 stimulation, HSC-enriched $\mathrm{LKS}^{+}$cells exhibited an even more prominent response than myeloid progenitors (Figure 4D), as indicated by intracellular phosphorylation of Stat6 (Figure 4E) which has been recognized as a downstream transducer of IL-4 signaling..$^{40}$ In response to exposure to IL-4, all MK-associated transcription factors except for Gata2 were universally downregulated in $\mathrm{LKS}^{+}$cells (Figure 4f), suggesting the possible effects of this cytokine on MK differentiation of primitive hematopoietic cells. We next analyzed the transcriptome of $\mathrm{LKS}^{+}$cells from AML BM $\left(_{\text {GSE52506) }}^{10}\right.$ and found significant upregulation of IL-4 signaling genes and predicted Stat6-bound genes (Figure $4 \mathrm{Gg})$. As BM immune cells have been reported to be the main source of IL-4, ${ }^{41}$ we first quantified the IL-4 mRNA expression in $\mathrm{T}$ lymphocytes, B lymphocytes, monocytes, macrophages, natural killer cells and eosinophils. However, we did not detect upregulation of IL-4 in these cells from AML BM (Online Supplementary Figure S6A). The level of expression of IL-4 by AML blasts was similar to that by normal hematopoietic cells (Online Supplementary Figure S6B). We then quantified IL-4 expression in HSC niche cells including mesenchymal stem cells, endothelial cells and osteoblasts. We detected reproducible significant upregulation of IL-4 in endothelial cells but not in the other niche cells (Figure 4H). Thus, BM endothelial cells produced excessive IL-4 in AML to activate IL-4 signaling in $\mathrm{HSC}$-enriched $\mathrm{LKS}^{+}$subsets.

To further understand the effects of IL-4 on MK differentiation, we intraperitoneally injected IL-4 into wildtype mice at $48 \mathrm{~h}$ intervals and analyzed the alterations of MK as well as HSPC with MK potential in mice BM (Figure $5 \mathrm{~A})$. In order to prolong the half-life of IL- 4 and enhance its biological activity in vivo, we pre-associated the IL-4 with its specific monoclonal antibody as previously describe ${ }^{42}$ and injected the IL-4 complex (IL-4cx) into the mice. We observed a remarkable decrease of platelets in peripheral blood after the injection of two doses, while erythrocytes and leukocytes were barely affected (Figure 5B). Simultaneously, though the total number of BM cells was not changed (Figure $5 \mathrm{C}$ ), the number of MK in the BM of IL-4-treated mice was severely reduced by $84 \%$ (Figure $5 \mathrm{D})$. The ploidy distribution of MK exhibited a left shift with high-ploidy $(\geq 32 \mathrm{~N})$ cells being more severely decreased (Figure 5E), indicating that IL-4 treatment suppressed MK maturation. Among HSPC with MK potential, we observed an $~ 54 \%$ loss of PreMegE and $~ 37 \%$ loss of MkP (Figure 5F), whereas the proportion of LT-HSC was unaltered, and that of MPPs increased $\sim 4$ fold (Figure 5G). These results suggest that the downward differentiation of MPP2 was severely hampered by IL-4, especially in the route via PreMegE. As expected, vWF expression was significantly reduced in MPP2 from IL-4-treated mice (Figure $5 \mathrm{H}$, I). Interestingly, we observed a remarkable increase of $\mathrm{vWF}^{+}$cells among LT-HSC (Figure 5H, I), indicating that IL-
4 did not have an obvious inhibitory effect on their MK differentiation in our setting; on the contrary, LT-HSC gave rise to $\mathrm{MK}$ more actively as compensation for the reduced contribution of MPP2 to the MK pool, which limited the loss of MkP to a relatively small extent. Given the smaller reduction $(\sim 37 \%)$ of $\mathrm{MkP}$ and the considerable decrease of MK ( 84\%) (Figure 5D, F), thrombocytopenia induced by IL-4 administration was largely caused by a drastic inhibition of MkP maturation. Notably, IL-4 receptor (IL-4R $\alpha$ ) expression on MPP2 and MkP was higher than that on LTHSC and PreMegE subsets (Figure 5J), in accordance with the more prominent response of MPP2 and MkP to in vivo IL-4 treatment.

\section{Targeting interleukin-4 in conjunction with chemotherapy enhances platelet recovery in acute myeloid leukemia mice}

Lastly, we tested whether targeting IL-4 could ameliorate thrombocytopenia in AML mice. To do this, AML mice were treated with anti-mIL-4 on days 7, 9, and 11 after injection of leukemic cells and were sacrificed on day 13 for analysis (Online Supplementary Figure S7A). Administration of anti-mIL- 4 alone to leukemic mice neither significantly decreased leukemia load nor increased platelet count in the peripheral blood (Online Supplementary Figure $S 7 B, C$ ). Intensive induction chemotherapy is currently used for patients with AML as a general therapeutic strategy. ${ }^{43,44}$ Because cytarabine (AraC) together with an anthracycline remains the mainstay of induction therapy, ${ }^{44}$ we set up a treatment protocol in which AML mice were treated daily with $60 \mathrm{mg} / \mathrm{kg}$ of $\mathrm{AraC}$ for 1 week (Online Supplementary Figure S7D). This treatment significantly reduced the leukemic burden in the peripheral blood (Online Supplementary Figure S7E) and prolonged the survival of AML mice (Online Supplementary Figure S7F), but it was noted that the animals developed severe thrombocytopenia (Online Supplementary Figure S7G). To test the hypothesis that thrombocytopenia may be more mitigated by anti-IL-4 given during or after AraC treatment (Online Supplementary Figure S7H), we next established a treatment protocol in which AML mice were intraperitoneally injected daily with $10 \mathrm{mg} / \mathrm{kg}$ of anti-mIL-4 during AraC chemotherapy (Figure 6A). Interestingly, this treatment not only significantly reduced the leukemic burden (Figure 6B) but also enhanced the recovery of platelets ( $\sim 4.7$-fold increase) and erythrocytes ( 1.9-fold increase). In contrast, leukocytes (Figure 6C) and serum thrombopoietin concentration (Online Supplementary Figure S7I) were not significantly affected. As a result, this new strategy of combining AraC with anti-IL-4 also significantly extended the duration of remission of AML mice (Figure 6D). Together, these data demonstrate that anti-mIL-4 combined with chemotherapy could improve the therapeutic response compared to that achieved with standard chemotherapy for AML.

\section{Discussion}

Thrombocytopenia is a frequent complication among AML patients: It can lead to a strong dependence on platelet transfusions and even fatal bleeding. Using an MLL-AF9-induced AML mouse model, we demonstrated that thrombocytopenia in AML was accompanied by a progressive loss of mature $\mathrm{MK}$ in the BM. A systematic 
A

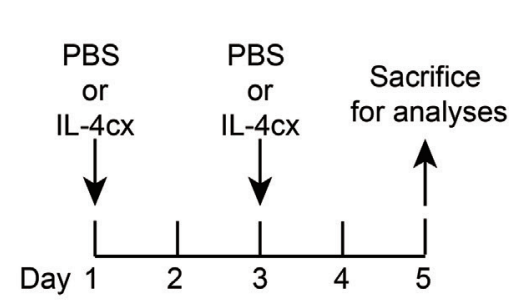

C

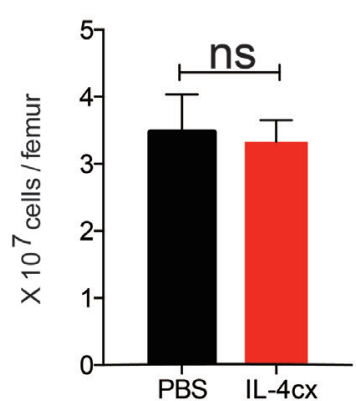

E

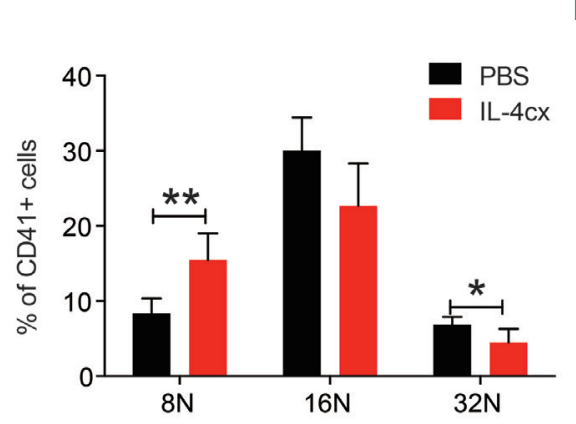

D

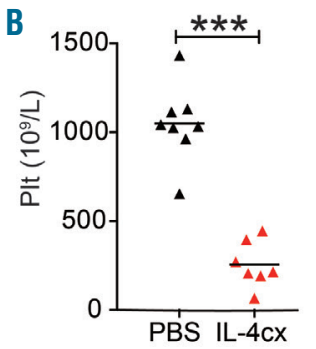

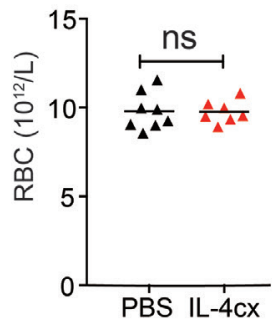

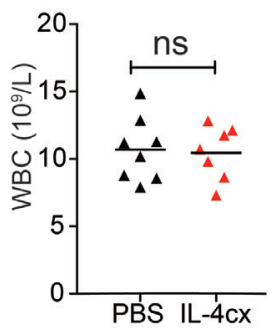

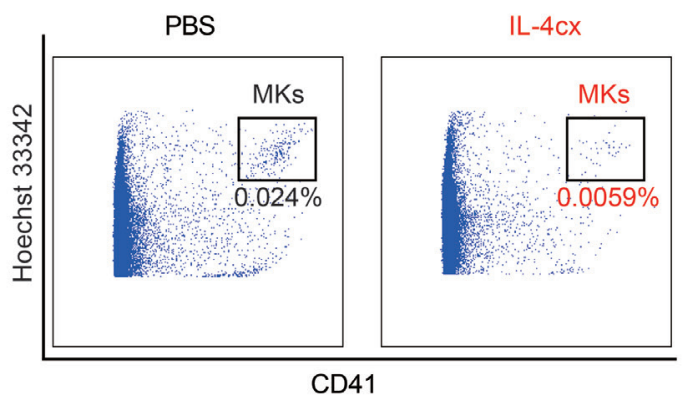
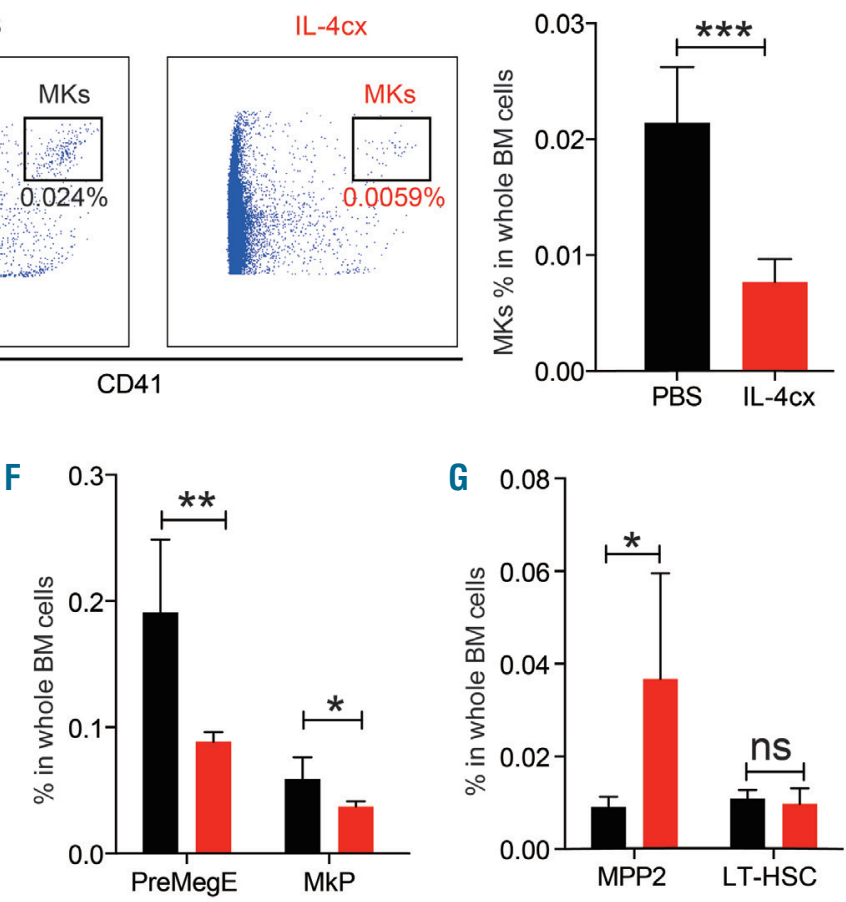

H
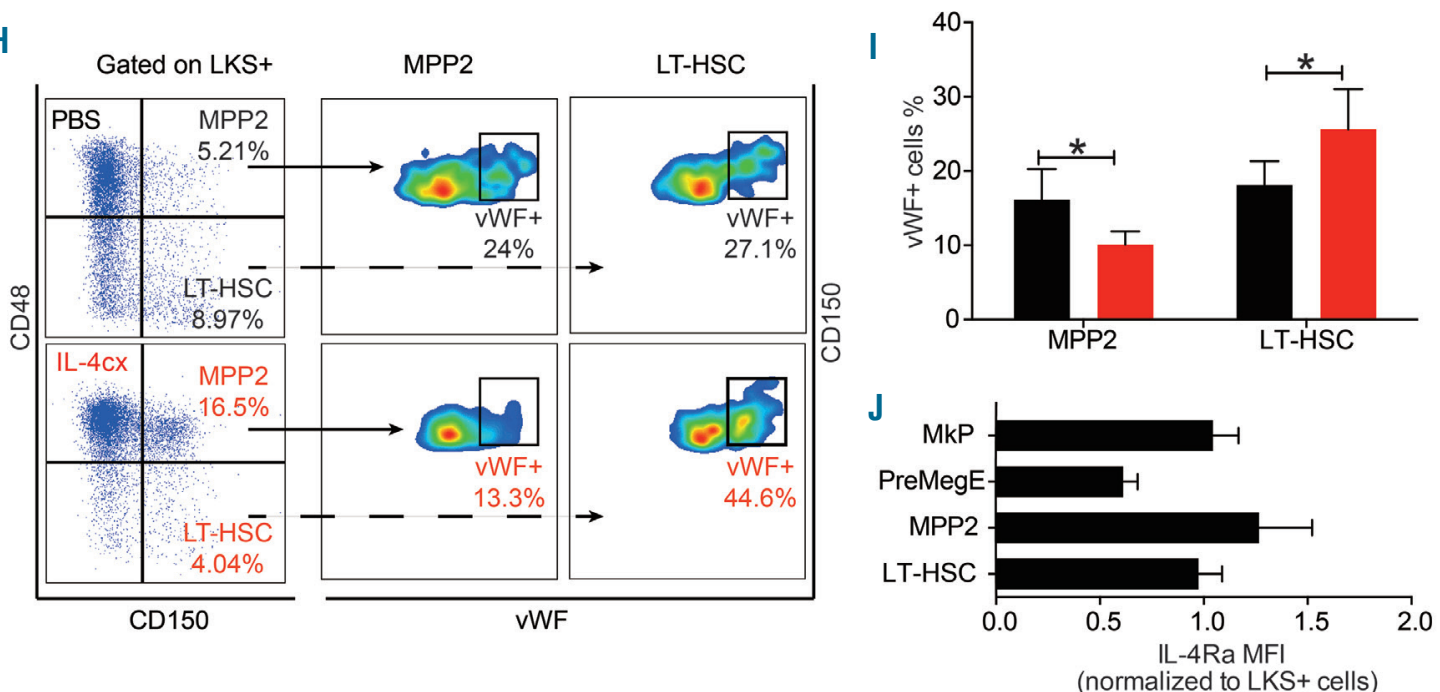

Figure 5. Inhibitory effects of interleukin-4 on megakaryopoiesis and thrombopoiesis in vivo. (A) Diagram of the experimental design: each mouse received intraperitoneal injections of $2 \mu \mathrm{g}$ murine interleukin-4 (IL-4) complexed (cx) with $10 \mu \mathrm{g}$ anti-mIL-4 (clone 11B11) or equivalent phosphate-buffered saline (PBS) on day 1 and day 3. Mice were sacrificed on day 5 for subsequent analyses. (B) Counts of platelets (PIt), erythrocytes (RBC) and leukocytes (WBC) in peripheral blood of mice injected with PBS or IL-4 complex (IL-4cx). Seven to eight mice per group; three independent experiments. (C) Total number of whole bone marrow cells per femur in mice injected with PBS or IL-4cx. Five mice per group; three independent experiments. (D) Representative flow cytometric plots (left) and percentage (right) of megakaryocytes (MK) in PBS or IL-4treated mice bone marrow. Five mice per group, three independent experiments. (E) Percentages of MK with different ploidy among CD41 ${ }^{+}$cells in the bone marrow of mice treated with PBS or IL-4. Five mice per group, three independent experiments. (F, G) Percentages of pre-megakaryocytic-erythroid (PreMegE) (F), megakaryocyte progenitors (MkP) (F), long-term hematopoietic stem cells (LT-HSC) (G) and multipotent progenitors (MPP2) (G) in the bone marrow of mice treated wih PBS or IL-4. Five mice per group, three independent experiments. $(\mathrm{H}, \mathrm{I})$ Representative flow cytometric plots $(\mathrm{H})$ and percentages (I) of von Willebrand factor (vWF) ${ }^{+}$cells in LT-HSC and MPP2 in the bone marrow from mice treated with PBS or IL-4. Five mice per group, three independent experiments. (J) Mean fluorescence intensity (MFI) of IL4R $\alpha$ on HSPC subsets, normalized to values in Lin/c-Kit ${ }^{+} / \mathrm{Sca}-1^{+}\left(\mathrm{LKS}^{+}\right)$cells. Four mice per group, two independent experiments. ${ }^{*} P<0.05, * * P<0.01, * * * P<0.001$. ns, no significant difference. Error bars represent the standard error of mean. 
A

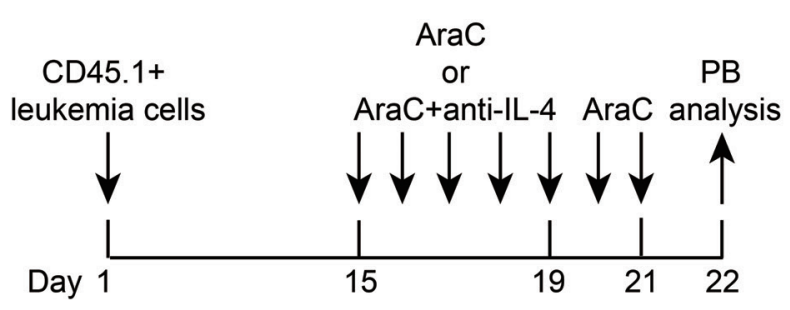

C
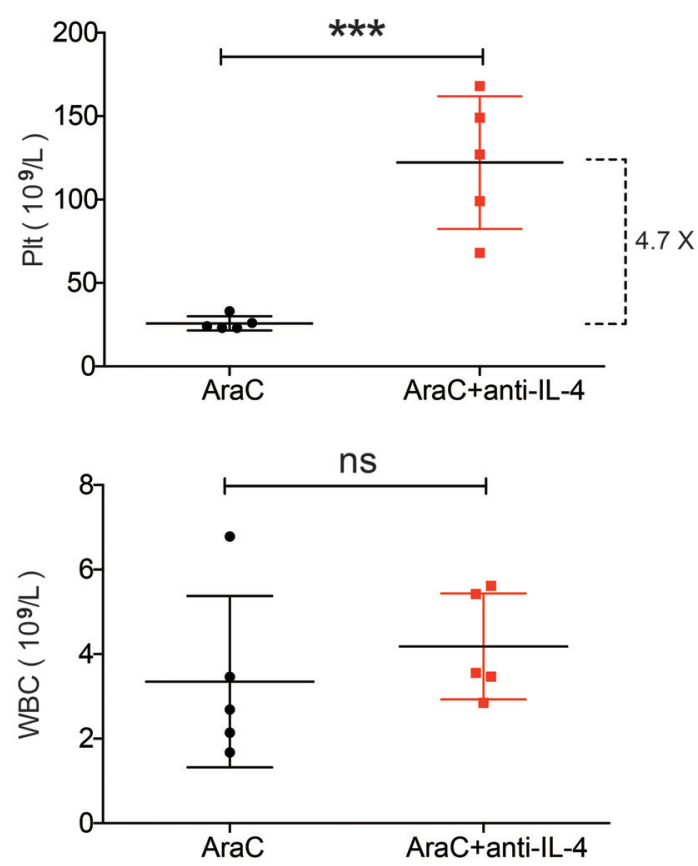

D
B
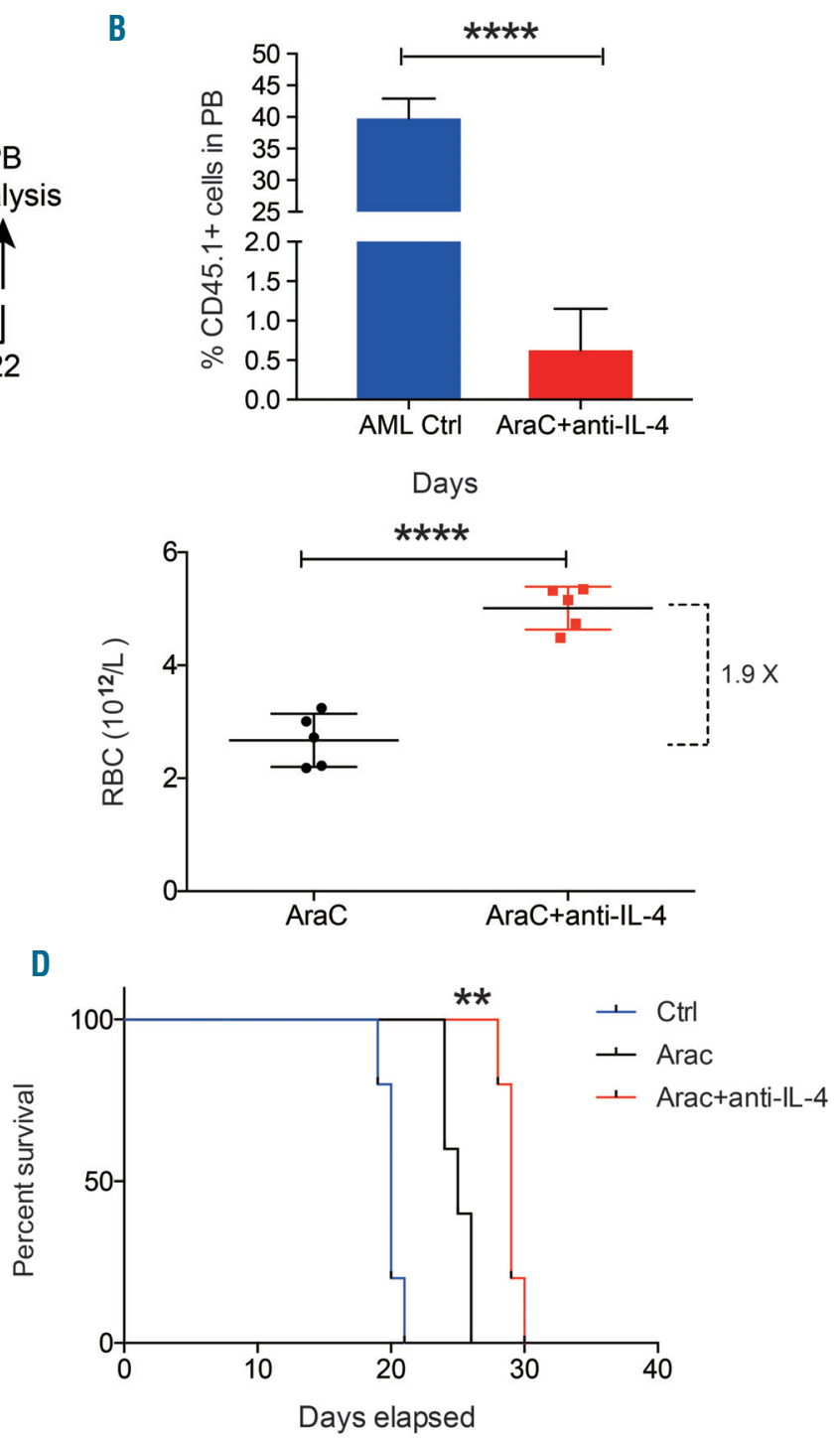

Figure 6. Targeting interleukin-4 cooperates with chemotherapy to enhance platelet recovery in acute myeloid leukemia mice. (A) Diagram of the experimental design: mice transplanted with CD45.1+ leukemic cells were treated with cytarabine (AraC) (60 mg/kg/day) for 1 week from day 15 , with or without anti-interleukin4 (anti-IL-4) $(10 \mathrm{mg} / \mathrm{kg} /$ day) for 5 days. The peripheral blood (PB) analysis was performed on day 22. (B) Percentage engraftment of CD45.1 leukemic cells in mice untreated (AML Ctrl) or treated as in (A): engraftment assessed in the PB. Five mice per group, three independent experiments. (C) Counts of platelets (PIt), erythrocytes (RBC) and leukocytes (WBC) in PB of mice injected with AraC alone or combined with anti-mIL-4. Five mice per group, three independent experiments. (D) Survival curves of recipient mice given different treatments as shown in (A). Five mice per group, three independent experiments. ${ }^{\circ} P<0.05, * \star P<0.01, * \star \star P<0.001$, $* * * * P<0.0001$. ns, no significant difference. Error bars represent the standard error of mean.

comparison of the megakaryocytic differentiation landscape between leukemia and healthy control mice revealed a marked reduction of $M K$ differentiation from MPP2 and LT-HSC via both the canonical and non-canonical pathways, which was fundamentally responsible for the decreased MK in AML BM. Transcriptome analysis of MK from animals with AML showed severely impaired maturation and platelet-producing capacity. Additionally, we discovered an excessive production of IL-4 by BM endothelial cells and found that this had a striking role in suppressing MK differentiation from MPP2 and MK maturation in vivo, which might contribute to the thrombocytopenia of mice with AML. Finally, our preclinical data using pharmacological approaches to inhibit IL-4 in combination with AraC treatment showed that targeting IL-4 represents a promising strategy to improve the therapeutic responses in AML.

By performing colony assays on whole BM cells, Capitano et al..$^{45}$ recently found that IL-4, in synergy with transforming growth factor- $\beta 1$, could inhibit hematopoietic progenitor cell colony formation. Our results further demonstrated that IL-4 had a unique inhibitory effect on colony-forming unit-MK formation without affecting other lineages, which was consistent with a previous study that assessed human $\mathrm{CD} 34^{+}$cells. ${ }^{37} \mathrm{NF}-\mathrm{E} 2$ transcription factor has been identified as an essential factor for terminal MK maturation and normal platelet production. ${ }^{46}$ It has been shown that IL-4 downmodulated the expression of NF-E2 transcription factor at both the mRNA and protein levels in normal maturing megakaryocytic cells 
and in megakaryoblastic cell lines. ${ }^{47}$ This suggests that the NF-E2 transcription factor might participate in the molecular mechanism of IL-4-induced defective MK differentiation and polyploidization in AML, but this requires further investigation.

Despite the marked response of $\mathrm{LKS}^{+}$cells to in vitro IL4 stimulation, IL-4 appeared to act to a greater degree on the last step of MK differentiation, rather than on LTHSC. Studies showed that MK could originate directly from an upstream HSC subpopulation, independently of other lineage fates. ${ }^{2,1,22}$ Therefore, the increase of $\mathrm{vWF}^{+} \mathrm{LT}$ HSC in IL-4-treated mice was more likely to be a compensation for MK reduction. In our AML mouse model, the number of PreMegE was dramatically reduced, while the decrease of MkP was less marked, which suggests that HSC compensated for the deficiency of MkP through the non-canonical route. In addition, the increase of $\mathrm{vWF}^{+}$ LT-HSC in IL-4-treated mice was consistent with the phenomenon observed in MK-depleted mouse models, ${ }^{48}$ suggesting the role of $\mathrm{vWF}^{+}$LT-HSC as MK reserves in native hematopoiesis and their relative resistance to stimuli. Thus, the blocked differentiation of LT-HSC in AML BM appears to result from a complex of factors, rather than IL-4 alone, including signals from niche cells, which require intensive research since they cannot be corrected easily by conventional cytotoxic therapy.

Studies have shown that leukemic cells impair the function of normal hematopoiesis by causing a significant change in a variety of niche cells and secreting cytokines in the BM microenvironment. ${ }^{3,69}$ In our study, the administration of IL-4 inhibitors alone to leukemic mice did not increase platelet counts in the peripheral blood, likely due to the absence of a specific effect of IL-4 inhibitors on leukemic cells. Currently the standard treatment of leukemia is primarily chemotherapy ${ }^{43}$ It has been reported that AML patients given induction treatment with highdose cytarabine had a considerably longer period of thrombocytopenia and received significantly more platelet transfusions in comparison to patients in the intermediate-dose group. ${ }^{49}$ Thus, it is important to use hematopoietic growth factors to accelerate platelet recovery. In our study, anti-IL-4 was administered in combination with chemotherapy, with results suggesting that this has clinical potential for alleviating thrombocytopenia in AML. Additionally, anti-IL-4 has been shown to be safe when administered to patients with asthma, ${ }^{50}$ implying that it could be applied in the treatment of AML. In our current study, all the data were generated based on a specific AML model and it is unclear whether results obtained can be generalized to other types of AML. More studies are, therefore, needed to expand this paradigm to other types of leukemia and to explore whether our findings in the mouse model can be translated to human AML.

In summary, our present study systemically describes defective megakaryopoiesis from HSC in AML and for the first time identifies BM endothelial cell-derived IL-4 as a candidate factor responsible for thrombocytopenia and a potential therapeutic target, in conjunction with chemotherapy, in patients with AML.

\section{Acknowlegments}

We thank Rong Guo (Cnkingbio Company) for technical support. This work was supported by grants from the National Key Research and Development Program of China (2016YFA0100600, 2017YFA0103400); the National Natural Science Foundation of China (81421002, 81730006, 81430004, 81670106, 81870086, 8181101081); CAMS Initiative for Innovative Medicine (2017-I2M-3-009, 2016-I2M-1-017); and the CAMS Fundamental Research Funds for Central Research Institutes (2016GH3100001, 2018PT31005).

\section{References}

1. Hersh EM, Bodey GP, Nies BA, Freireich EJ. Causes of death in acute leukemia: a tenyear study of 414 patients from 1954-1963. JAMA. 1965;193:105-109.

2. Chang HY, Rodriguez V, Narboni G, Bodey GP, Luna MA, Freireich EJ. Causes of death in adults with acute leukemia. Medicine (Baltimore). 1976;55(3):259-268.

3. Cheng H, Cheng T. 'Waterloo': when normal blood cells meet leukemia. Curr Opin Hematol. 2016;23(4):304-310.

4. Lichtman MA. Interrupting the inhibiton of normal hematopoiesis in myelogenous leukemia: a hypothetical approach to therapy. Stem Cells. 2000;18(5):304-306.

5. Morrison SJ, Scadden DT. The bone marrow niche for haematopoietic stem cells. Nature. 2014;505(7483):327-334.

6. Colmone A, Amorim M, Pontier AL, Wang S, Jablonski E, Sipkins DA. Leukemic cells create bone marrow niches that disrupt the behavior of normal hematopoietic progenitor cells. Science. 2008;322(5909):1861-1865.

7. Zhang B, Ho YW, Huang Q, et al. Altered microenvironmental regulation of leukemic and normal stem cells in chronic myeloge- nous leukemia. Cancer Cell. 2012;21(4):577592.

8. Hoggatt J, Kfoury Y, Scadden DT. Hematopoietic stem cell niche in health and disease. Annu Rev Pathol. 2016;11:555-581.

9. Sanchez-Aguilera A, Mendez-Ferrer S. The hematopoietic stem-cell niche in health and leukemia. Cell Mol Life Sci. 2017;74(4):579590.

10. Cheng H, Hao S, Liu Y, et al. Leukemic marrow infiltration reveals a novel role for Egr3 as a potent inhibitor of normal hematopoietic stem cell proliferation. Blood. 2015;126 (11):1302-1313.

11. Miraki-Moud F, Anjos-Afonso F, Hodby KA, et al. Acute myeloid leukemia does not deplete normal hematopoietic stem cells but induces cytopenias by impeding their differentiation. Proc Nati Acad Sci U S A. 2013;110(33):13576-13581.

12. Wang Y, Gao A, Zhao H, et al. Leukemia cell infiltration causes defective erythropoiesis partially through MIP-1alpha/CCL3. Leukemia. 2016;30(9):1897-1908.

13. Mukherjee S. "Blood feuds". Blood. 2015;126(11):1264-1265.

14. Colby-Graham MF, Chordas C. The childhood leukemias. J Pediatr Nurs. 2003;18 (2):87-95.
15. Webert K, Cook RJ, Sigouin CS, Rebulla P Heddle NM. The risk of bleeding in thrombocytopenic patients with acute myeloid leukemia. Haematologica. 2006;91(11): 1530-1537.

16. de Sauvage FJ, Carver-Moore K, Luoh SM, et al. Physiological regulation of early and late stages of megakaryocytopoiesis by thrombopoietin. J Exp Med. 1996;183(2):651-656.

17. Rauch PJ, Ellegast JM, Widmer CC, et al. MPL expression on AML blasts predicts peripheral blood neutropenia and thrombocytopenia. Blood. 2016;128(18):2253-2257.

18. Kuter DJ, Begley CG. Recombinant human thrombopoietin: basic biology and evaluation of clinical studies. Blood. 2002;100 (10):3457-3469.

19. Passaro D, Tullio AD, Abarrategi A, et al. Increased vascular permeability in the bone marrow microenvironment contributes to disease progression and drug response in acute myeloid leukemia. Cancer Cell. 2017;32(3):324-341.

20. Akashi K, Traver D, Miyamoto T, Weissman IL. A clonogenic common myeloid progenitor that gives rise to all myeloid lineages. Nature. 2000;404(6774):193-197.

21. Rodriguezfraticelli AE, Wolock SL, Weinreb $\mathrm{CS}$, et al. Clonal analysis of lineage fate in 
